\title{
Eucalyptus globulus Oil Versus Bleach Use as Antiseptic Agents in Podoconiosis Treatment: An Interventional Study in Wolaita Zone, Southern Ethiopia
}

\author{
Bereket Alemayehu $^{1,}$, Terefe Gelibo², Zewde Zema Kanche ${ }^{3}$ \\ ${ }^{1}$ Biomedical Science Division, Biology Department, College of Natural and Computational Sciences, Wolaita Sodo University, Wolaita \\ Sodo, Ethiopia \\ ${ }^{2}$ Health System Research Directorate, Ethiopian Public Health Institute, Addis Ababa, Ethiopia \\ ${ }^{3}$ Department of Pharmacy, College of Health Sciences and Medicine, Wolaita Sodo University, Wolaita Sodo, Ethiopia
}

\section{Email address:}

bereketalemayehu@gmail.com (B. Alemayehu),mamater.1986@gmail.com (T. Gelibo), zedzem@gmail.com (Z. Z. Kanche)

${ }^{*}$ Corresponding author

\section{To cite this article:}

Bereket Alemayehu, Terefe Gelibo, Zewde Zema Kanche. Eucalyptus globulus Oil Versus Bleach Use as Antiseptic Agents in Podoconiosis Treatment: An Interventional Study in Wolaita Zone, Southern Ethiopia. American Journal of Biomedical and Life Sciences. Vol. 5, No. 6, 2017, pp. 113-118. doi: 10.11648/j.ajbls.20170506.11

Received: June 5, 2017; Accepted: June 19, 2017; Published: November 8, 2017

\begin{abstract}
Eucalyptus globulus essential oil is widely used for medicinal purpose because of its pharmacological constituents. The present study aimed to use water emulsified E. globulus oil as an antiseptic agent in the treatment protocol of podoconiosis (non-filarial elephantiasis) and compare disease improvement upon E. globulus oil versus bleach (usual antiseptic chemical) use in the treatment protocol. An interventional study was conducted from January to March, 2012 to compare disease improvement on podoconiosis upon using water emulsified $E$. globulus oil versus bleach-water solution as antiseptic agents in podoconiosis treatment. Forty podoconiosis patients purposely selected and randomly allocated into intervention $(E$. globulus oil) group and comparison (bleach) group with matched clinical stage of podoconiosis. After the baseline data recording, home based treatment was started and follow-up had been undertaken every week for 3-month period. The data were analyzed using SPSS version 16 statistical package. The mean clinical stages of the disease among the study participants were 2.72 for the right and 2.80 for the left legs before the treatment start. Seventeen (32.5\%) of patients suffered from the disease for more than 25 years. For all the outcomes measured at the end of study period, the individual treatment effects of eucalyptus oil and bleach antiseptic agents were compared and the result showed no significant difference. Hence, eucalyptus oil emulsified in water can be used as alternative antiseptic agent in podoconiosis treatment protocol.
\end{abstract}

Keywords: Eucalyptus Oil, Podoconiosis, Bleach, Wolaita, Ethiopia

\section{Introduction}

Plants have been used for treatment of different diseases in human history before the appearance of modern clinical drugs. The essential oils extracted from different parts of these plants are known to contain active agents that can be used for therapeutic purposes or as precursors for the synthesis of useful drugs [1]. Essential oils extracted through steam distillation are used as antimicrobial agents due to their chemical composition [2]. The functional groups of some compounds found in most plant materials such as alcohol, phenols, terpenes and ketones are associated for antimicrobial characteristics [3, 4].

Medical properties of eucalyptus have been known to the global community since several years ago and have now become common and useful plant in many tropical regions with all its benefits [5]. Leave extract of eucalyptus essential oil has been traditionally used to heal wounds and microbial infections $[5,6]$. In multiple experimental pharmacologic studies, eucalypti had shown antimicrobial activities $[7,8,9]$, and anti-inflammatory activity [10]. Eucalyptus oil also had shown analgesic activity in a clinical pharmacologic study [11]. Even though there is no efficacy study in patients, 
eucalyptus extracts with clean cooking oil was traditionally used for skin care in podoconiosis patients in some areas of Ethiopia [12, 13].

Podoconiosis is an endemic non-filarial elephantiasis which is widely distributed in the world with its highest prevalence in tropical or sub tropical latitudes [14]. The distribution of the disease is associated with the presence of irritant red clay soil of volcanic origin in the environment. The affected individuals are mainly bare footed farmers that work on red clay soils [15]. The disease has different stages in its pathology with different clinical manifestations. As the most risk group among the community are poor bare-footed farmers who are not well educated, awareness creation through education on control and prevention methods should be the priority area in the disease management programs. Habit of maintaining foot-care and hygiene are one of the forefront management techniques in the treatment and prevention of podoconiosis [16]. It is commonly understood that microorganisms ideally grow in unhygienic wounds of elephantoid feet and further complicate the disease by developing infections [17, 18]. The treatment protocol of podoconiosis was adopted from that of filarial elephantiasis [17] and it constitutes 1) washing feet with soap and water, 2) antiseptic use, 3) drying legs with clean towel/cloth, 4) applying topical ointment, 5) elevation of elephantoid leg/s and 6) consistent use of socks and shoes. A chemical agent currently being used widely as an antimicrobial agent in podoconiosis treatment procedure is sodium hypochlorite (bleach). Even though bleach is currently an option of use in the treatment of the disease because of its potential microbicidal action, it may have negative health effect upon long period of exposure [19]. Therefore, other alternative agents should be sought. The current study was conducted to observe the disease improvement after using naturally bioactive extract, E. globulus essential oil, as an antiseptic agent in the foot-care procedure during podoconiosis treatment. This study was also aimed to compare effects of the two antiseptic agents, E. globulus essential oil versus bleach, based on the disease improvement after treatment.

\section{Materials and Methods}

\subsection{Study Area}

The study was conducted in Zamine Nare Kebele (the smallest administrative unit) of Damot Sore Woreda, Wolaita Zone, Southern Ethiopia. Wolaita is located about $329 \mathrm{kms}$ south of Addis Ababa. According to Central Statistical Authority of Ethiopia report [20], in the zones over 95\% of the population lives on subsistent farming in which almost all farmers don't have the habit of wearing shoe during farm work and hence are exposed to soil sourced disease like podoconiosis [21]. Wolaita Zone is known to be an endemic area for podoconiosis in southern Ethiopia [18]. In the Zone, the prevalence of podoconiosis is more than 5\% [22]. A NonGovernmental Organization, Mossy Foot International (MFI), is almost a sole community-based organization which offers prevention and treatment for podoconiosis patients at 15 main outreach sites. Gununo Mossy Foot outreach treatment site is the nearest treatment site to Zamine Nare Kebele, the present study locality.

\subsection{Selection of Patients}

Podoconiosis patients who had resided for more than 10 years in the study area were selected by conducting a houseto-house morbidity survey to include each type of the five clinical stages of the disease at Zamine Nare village of Damot Sore in Wolaita Zone. These patients were registered in the registry book of Gununo Mossy Foot outreach site, one of the outreach sites of Mossy Foot International (MFI) project in Wolaita Zone, for future follow-up after this study. The patients were asked for history and examined for disease condition by the community health workers of MFI who had experiences in clinical examination, treatment and prevention of podoconiosis. On the first presentation, each patient's demographic data including age, sex and baseline parameters such as clinical stage, presence $(\mathrm{M}+)$ or absence $(\mathrm{M}-)$ of mossy changes, below-knee leg circumference, presence or absence of wound and its smell were recorded for both right and left legs of each patient.

\subsection{Study Design and Patient Recruitment}

An interventional study was conducted from January to March, 2012 to compare disease improvement on podoconiosis upon using E. globulus oil and bleach solutions as antiseptic agents in the treatment protocol of the disease. Forty podoconiosis patients ( 25 female and 15 male patients) between the ages of 18 and 70 years, (mean 49 years), who had given verbal informed consent, were purposely selected and categorized into two groups based on their clinical stage of podoconiosis. The clinical staging of podoconiosis used in this study was adopted from a staging system developed by Tekola and others [18]. Patients were randomly allocated into treatment and comparison groups, E. globulus oil group and bleach group. Both groups therefore contained 20 patients with matching disease stage.

E. globulus-oil group (treatment group): This group contained podoconiosis patients who received all the usual treatment modalities except the bleach-water dilution. For this group, E. globulus oil- water emulsified solution was given as antiseptic agent replacing the usual Bleach-water solution. $5 \mathrm{ml}$ of E. globulus oil was added into approximately $5 \mathrm{~L}$ of lukewarm water in a bucket and mixed well manually using stirrer. After emulsifying the oil in water, patients immersed their legs in oil-in-water emulsion for about 20 minutes.

Bleach group (comparison group): In this group, podoconiosis patients received all the usual treatment modalities in which $5 \mathrm{ml}$ of bleach in $5 \mathrm{~L}$ of water is used as an antiseptic agent.

\subsection{Follow-up and Outcome Evaluation}

After recording the baseline data at Zamine Nare Health 
Center, home based treatment was started immediately on the first day of the study. Follow-up was then undertaken every week for 3-month period. Outcome measures used for monitoring the disease progress were done in reference to the clinical staging system which was developed by Tekola and others [18] for podoconiosis. The Tekola clinical staging system for podoconiosis incorporates three clinical measures - numerical stage (1 to 5), presence $(\mathrm{M}+$ ) or absence (M2) of mossy skin changes, and a measurement (in $\mathrm{cm}$ ) of the widest below-knee circumference. In addition to the mentioned clinical measures, presence and absence of wound and foot smell were included as additional outcome measures. All measures were recorded blind to the previous measurements by the same team of community health workers of Mossy Foot International.

The outcome measures recorded at the beginning and end of the study period were compared for eucalyptus oil and bleach treatment groups and then the disease improvement was assessed on the following parameters: improvement on clinical stages, reduction of leg circumference, mossy reduction, skin texture, acute attack (acute adenolymphangitis) reduction and bad odor disappearance. These results are only commented in the discussion. There should be a paragraph about them in the results section once that these observations are cited in materials and methods.

\subsection{Statistical Analysis}

The treatment and patient characteristic data were analyzed using SPSS version 16 statistical package. The disease conditions of the two groups were compared at baseline using the t-test for continuous variables or Pearson Chi square tests for discrete variables. An independent-samples t-test was performed to compare treatment outcomes between the two groups. Proportions, percentages, tables and graphs were used to summarize the collected data accordingly. A $p$ value of $>$ 0.05 was considered statistically insignificant.

\subsection{Ethics Statements}

The protocol of this study was ethically reviewed and approved by ethical review committee of Collage of Natural and Computational Science of Wolaita Sodo University. Permissions were obtained from Wolaita Zone Health Department and Damot Sore Woreda Health Bureau. Verbal informed consent was also obtained from podoconiosis patients. Since most of the study participants were not able to read and write, verbal consent was obtained from each study participant. This use of verbal consent was approved by the ethical review committee. The objective of the study was explained to the patients. Name and other identifying information were not mentioned in the study. Participants were informed that the information gathered in this study would be kept confidential and they had full rights to participate or not in the study. Permission was obtained from patients to present and disseminate the study finding whenever needed. At the end of this follow-up study, all study subjects were linked to Gununo Mossy Foot outreach site to receive the usual treatment and get further care in the future.

\section{Results}

\subsection{Socio-demographic and Baseline Disease Conditions of the Study Participants}

Among 40 individuals, 15 (37.5\%) males and 25 (62.5\%) were females. The majority $(37.5 \%)$ of participants were housewives followed by farmer $32.5 \%$. Majority $(87.5 \%)$ of the study participants had never attended any formal education. The mean age of participants was found to be $46.92(\mathrm{SD} \pm 11.5)$ and mean year of patients lived with the disease was $19.62(\mathrm{SD} \pm 12.39)$ (Table 1$)$.

Table 1. Socio-demographic Characteristics and Disease Conditions of Study Participants at Baseline.

\begin{tabular}{|c|c|c|c|c|}
\hline Variables & & Eucalyptus treated & Bleach treated & Overall ( $\%$ or Mean) \\
\hline Mean age \pm SD & & $43.5 \pm 8.2$ & $50.15 \pm 13.6$ & $46.92 \pm 11.5$ \\
\hline \multirow{2}{*}{$\operatorname{Sex}(n=40)$} & Male & 10 & 5 & $15(37.5 \%)$ \\
\hline & Female & 10 & 15 & $25(62.5 \%)$ \\
\hline \multirow{4}{*}{ Occupation } & House wife & 9 & 4 & $15(37.5 \%)$ \\
\hline & Farmer & 3 & 11 & $13(32.5 \%)$ \\
\hline & Potter & 3 & 4 & $7(17.5 \%)$ \\
\hline & Merchant & 5 & 1 & $5(12.5 \%)$ \\
\hline \multirow{2}{*}{ Educational status } & No formal education & 19 & 16 & $35(87.5 \%)$ \\
\hline & At least primary level & 1 & 4 & $5(12.5 \%)$ \\
\hline \multirow{2}{*}{ Marital status } & Married & 17 & 19 & $36(90 \%)$ \\
\hline & Widowed & 3 & 1 & $4(10 \%)$ \\
\hline \multirow{5}{*}{ Stage of the disease } & Stage 1 & 2 & 2 & $4(10.0 \%)$ \\
\hline & Stage 2 & 6 & 6 & $12(30.0 \%)$ \\
\hline & Stage 3 & 6 & 6 & $12(30.0 \%)$ \\
\hline & Stage 4 & 4 & 4 & $8(20.0 \%)$ \\
\hline & Stage 5 & 2 & 2 & $4(10.0 \%)$ \\
\hline \multirow{5}{*}{ Acute attach frequency } & Every week & 2 & 2 & $4(10.0 \%)$ \\
\hline & Every two weeks & 5 & 6 & $11(27.5 \%)$ \\
\hline & Every month & 11 & 8 & $19(47.5 \%)$ \\
\hline & Every 3 months & 1 & 4 & $5(12.5 \%)$ \\
\hline & Every 6 months & 1 & 0 & $1(2.5 \%)$ \\
\hline
\end{tabular}




\begin{tabular}{lllll}
\hline Variables & & Eucalyptus treated & Bleach treated & Overall (\%o or Mean) \\
\hline & Hot \& dry season & 2 & 5 & $7(17.5 \%)$ \\
& Hot \& wet season & 3 & 2 & $5(12.5 \%)$ \\
Pain with weather & Cold \& dry season & 4 & 4 & $8(20.0 \%)$ \\
& Cold \& wet season & 7 & 5 & $12(30.0 \%)$ \\
& Not affected by weather & 4 & 4 & $8(20.0 \%)$ \\
& Morning & 3 & 4 & $7(17.5 \%)$ \\
Pain within a day & Noon & 6 & 7 & $13(32.5 \%)$ \\
& Evening & 8 & 6 & $14(35 \%)$ \\
& Night & 3 & 3 & $6(15 \%)$ \\
Year stayed with disease & $4-14$ & 10 & 7 & $17(42.5 \%)$ \\
& $15-24$ & 4 & 2 & $6(15 \%)$ \\
Mean year stayed with disease \pm SD & $25-34$ & 5 & 6 & $11(27.5 \%)$ \\
Treatment history & $34+$ & 1 & 5 & $6(15 \%)$ \\
& & $17.65 \pm 11.65$ & $21.60 \pm 13.08$ & $19.62 \pm 12.39$ \\
& Yes & 6 & 11 & $17(42.5)$ \\
\end{tabular}

Observations made at the beginning of the study showed a considerable complication of the disease among the study participants. Among the subjects, a significant number 24 $(60 \%)$ were greater or equal to the median clinical stage (3) of podoconiosis. The mean clinical stage of disease for the cohort was 2.76. Acute attach (acute inflammation of the foot) was happening for more than $95 \%$ of patients in every three month period with varying frequency. Twelve $(30 \%)$ of patients felt intense pain during cold and wet seasons of the years (Table 1).

\subsection{Foot Care Practices Among the Study Participants}

It was observed at the start of this study that only $8 \%$ of the subjects had feet clean and intact though almost all $(97.5 \%)$ of them responded as they had access to water to wash their feet. In the present study, $23(57.5 \%)$ of the patients had never worn shoes in their lifetime. There was inconsistent shoe wearing habit among 17 (42.5\%) of the patients. The most commonly owned shoes type was closed plastic. Among the patients that had inconsistent shoe wearing habit, $10(58.8 \%)$ reported to wear shoes for five days or more in a week while 7 (41.2\%) reported less than two days per week or less often than weekly.

\subsection{Changes in Outcome Variables Measured from Baseline to 3 Months}

After three months of treatment, it was observed that complications were reduced in both treatment groups with pooled improvement of mossy in $70.6 \%$, acute attach in $94.4 \%$, decrease by at least one clinical stage in $45 \%$ and decrease in circumference measure by at least one centimeter in $70 \%$ of patients. Bad odor was disappeared in both groups after treatment. Observations on the skin texture of the diseased legs and feet after treatment showed better improvement among patients in eucalyptus oil group.

Table 2. Changes in Mossy and Acute Attach Measured from Baseline to 3 Months.

\begin{tabular}{|c|c|c|c|c|c|c|c|}
\hline \multirow{2}{*}{ Variables } & \multicolumn{2}{|l|}{ At baseline } & \multicolumn{2}{|c|}{ After 3 months treatment } & \multicolumn{3}{|c|}{ Percent improvement after 3 months } \\
\hline & Eucalyptus & Bleach & Eucalyptus & Bleach & Eucalyptus & Bleach & Total \\
\hline Mossy present (\%) & $13(65 \%)$ & $4(20 \%)$ & $3(15 \%)$ & $2(10 \%)$ & $10(76.9 \%)$ & $2(50 \%)$ & $12(70.6 \%)$ \\
\hline Acute attach presence within 3 month (\%) & $10(50 \%)$ & $8(40 \%)$ & $1(5.0 \%)$ & 0 & $9(90 \%)$ & $0(100 \%)$ & $17(94.4 \%)$ \\
\hline
\end{tabular}

Table 3. Changes in Clinical Stage and Circumference Measured from Baseline to 3 Months.

\begin{tabular}{|c|c|c|c|c|c|c|c|c|c|}
\hline \multirow[b]{2}{*}{ Variables } & \multicolumn{3}{|l|}{ At baseline } & \multicolumn{3}{|c|}{ After 3 months' treatment } & \multicolumn{3}{|c|}{ Mean difference after 3 months } \\
\hline & Eucalyptus & Bleach & $\begin{array}{l}\text { P value } \\
\text { for } t \text { test }\end{array}$ & Eucalyptus & Bleach & $\begin{array}{l}\text { P value } \\
\text { for } t \text { test }\end{array}$ & Eucalyptus & Bleach & $\begin{array}{l}\text { P value } \\
\text { for t test }\end{array}$ \\
\hline Clinical stage mean & 2.75 & 2.78 & 0.941 & 2.13 & 2.18 & 0.863 & -0.63 & -0.60 & 0.880 \\
\hline Circumference mean $(\mathrm{cm})$ & 25.48 & 24.70 & 0.526 & 24.20 & 23.50 & 0.545 & -1.28 & -1.20 & 0.814 \\
\hline
\end{tabular}

\section{Discussion}

Podoconiosis, an endemic non-filarial elephantiasis of the lower leg, is affecting several people in tropical and subtropical highlands of the world. However, the disease has not yet gained much attention in the modern medication, though it has huge public health concern in the endemic areas. Hence, there are very limited treatment options exist for podoconiosis in the community-based treatment centers. The existing non-standardized treatment protocols of podoconiosis is simply shared, with some modification, from the treatment procedures of lymphoedema caused by lymphatic filariasis [17]. The local non-governmental organization, Mossy Foot International (MFI), has pioneered the adoption of this historic prevention and treatment protocol that is still being used for community-based interventions for podoconiosis in Ethiopia. The podoconiosis treatment protocol mainly focuses on the daily foot hygiene and the use of shoes, socks and elastic bandages [16].

There is no doubt that introducing any appropriate hygiene and skin care regimen in podoconiosis treatment and control 
will provide gains in the management of the disease. However, bringing such regimen into practice demands thorough investigations towards what has been and is being done in traditional and conventional medications to alleviate health problems under similar circumstances. In the present study, E. globulus essential oil emulsified in water was used as an antiseptic agent in the treatment protocol of podoconiosis Though there have been some traditional practices of using neem tree extracts and eucalyptus leaf soak for foot washing by podoconiosis patients in some parts of Ethiopia, the effect of use of E. globulus essential oil in podoconiosis treatment hasn't been studied so far.

The existing evidence from treatment-experience of Mossy Foot International indicated that effective prevention of podoconiosis can be achieved by regularly wearing shoes and washing feet from childhood. In early onset of the disease, foot hygiene is very helpful to halt and reverse the disease progress. It involves washing feet with soap and water, use of antiseptic and emollients, and consistently wearing shoes and socks [16]. However, in the present study, it was observed that majority of the study participants had unclean foot and hadn't owned a pair of shoes in their life. The reason for having unclean foot was not due to inaccessibility of water; but because of the absence of habit of regular foot washing. Lack of shoes in the present study participants was mainly because of inability to afford it.

In the present study, the study participants presented so many complications of which almost all showed improvement after three months follow-up. Both eucalyptus oil and bleach are potential antiseptic agents [23]. The outcome measures such as mossy foot, acute attach frequency, clinical stage of the disease, and circumference mean showed overall improvement in both eucalyptus oil and bleach groups. When the individual groups were compared based on changes in the outcome measures from 0 to 3 months, the analysis showed no significant difference on clinical stage of disease and mean circumference of the legs. Eventhough, eucalyptus group showed better improvement in general, there was no statistical difference seen in the t-test for the mean reduction in clinical stage in eucalyptus oil group (mean reduction $=0.63$ ) and bleach treatment group (mean reduction $=0.60)(p=0.880,95 \%$ CI -0.307 to 0.357$)$. The same was true for absence of statistical difference between the two groups for the mean reduction in leg circumference, 1.28 and 1.20 for eucalyptus and bleach respectively ( $\mathrm{p}=0.814,95 \%$ CI -0.565 to 0.715 ).

The observed improvement in foot and leg skin texture among patients in eucalyptus oil group with better skin smoothness and elasticity could be due to the effect of eucalyptus oil absorbed by the skin [24, 25]. Even though pain relief and anti-inflammatory conditions were not specifically studied in the present study, it was responded by participants in eucalyptus oil group that oil use resulted in better pain relief and anti-inflammatory effect. Supporting evidence for benefits of eucalyptus oil use for medical conditions were presented in other in-vivo and in-vitro studies $[10,26,27]$. The fragrance of eucalyptus oil was also liked by the study participants and their family, whereas the smell of bleach was hated so that patients were sometimes forced to apply the treatment in hidden places.

Having said the above discussion, the investigators of this study would like to disclose the limitations of the study. Even though this follow-up study had two groups, the test and the comparison groups, absence of untreated control group could be mentioned as a limitation. But, because the investigators found that it was ethically difficult to deny the affected individuals from accessing the regimen, inclusion of untreated control group was avoided. In the present study, only gross disease improvement was observed in both groups, eucalyptus oil versus bleach, rather than observing the single treatment effect of each agent in the treatment protocol.

\section{Conclusion}

The result of comparison of disease improvement after using eucalyptus oil and bleach antiseptic agents in the treatment protocol showed that the two groups came-up with comparative outcomes implying that eucalyptus oil emulsified in water might also be used as an antiseptic agent in the podoconiosis treatment. Further investigations are needed to increase the treatment options for podoconiosis. Researches should also be conducted to see the efficacy of individual treatment components used in the intervention of podoconiosis.

\section{References}

[1] Ayepola OO, Adeniyi BA (2008) The Antibacterial Activity of Leaf Extracts of Eucalyptus camaldulensis (Myrtaceae). J App Sci Res 4: 1410-1413.

[2] Cimanga K, Kambu K, Tona L, et al. (2002) Correlation between chemical composition and antibacterial activity of essential oils of some aromatic medicinal plants growing in the Democratic Republic of Congo. J Ethnopharmacol 2: 213220.

[3] Braca A, Siciliano T, D’Arrigo M, Germanò MP (2008) Chemical composition and antimicrobial activity of Momordica charantia seed essential oil. Fitoterapia 79:123125.

[4] Nair R, Vaghasiya Y, Chanda S (2008) Antibacterial activity of Eucalpytus citriodora Hk. oil on few clinically important bacteria. Afr J Biotechnol 7: 25-26.

[5] Adeniyi BA, Lawal TO, Olaleye SB (2006) Antimicrobial and gastro-protective activities of Eucalyptus camaldulensis (Myrtaceae) crude extracts. J Bio Sci 6: 1141-1145.

[6] Ranjit RR, Ajit RS, Bhagyashree BJ (2014) Antimicrobial activity of Azadirachta indica (Neem) against Pathogenic Microorganisms. JAIR 3:327-329.

[7] Brantner A, Grein E (1994) Antibacterial activity of plant extracts used externally in traditional medicine. J Ethnopharmacol 44:35-40.

[8] El-Keltawi NEM (1980) Antimicrobial activity of some Egyptian aromatic plants. Herba Polonica 26:245-250. 
[9] Janssen AM (1986) Screening for antibacterial activity of some essential oils by the agar overlay technique. Pharmaceutisch Weekblad 8:289-292.

[10] Wagner H (1986) In vitro inhibition of prostaglandin biosynthesis by essential oils and phenolic compounds. Planta Medica 3:184-187.

[11] Göbel H (1995) Essential plant oils and headache mechanisms. Phytomedicine 2:93-102.

[12] Mark L, Meskele A, Gail D (2010) Podoconiosis control guideline. Page 26.

[13] Molla YB, Davey G (2012) Podoconiosis control in rural Ethiopia: the roles of expert patients, appropriate treatment and community mobilization. Community Dermatology Page 3.

[14] Price EW (1976) The association of endemic elephantiasis of the lower legs in East Africa with soil derived from volcanic rocks. Trans R Soc Trop Med Hyg 70: 288- 295.

[15] Davey G, Tekola F, Newport MJ (2007) Podoconiosis: noninfectious geochemical elephantiasis. trans R Soc Trop Med Hyg 101(12):1175-1180.

[16] Davey G, Burridge E (2009) Community based control of a neglected tropical disease: the Mossy Foot Treatment and Prevention Association. PLoS Negl Trop Dis 3(5): e424.

[17] Dreyer G, Addiss D, Dreyer P, Noroes J (2002) Basic Lymphedema Management, first Ed. New Hampshire, US: Hollis Publishing Company Page 25.

[18] Tekola F, Ayele Z, Haile Mariam D, Fuller LC, Davey G (2008) Development and testing of a de novo clinical staging system for podoconiosis (endemic nonfilarial elephantiasis). Trop Med Int Health 13: 1277-1283.

[19] Incident Management and Toxicology (IMT) (2016) Sodium hypochlorite: health effects. Accessed at: https://www.gov.uk access date: $21 / 1 / 2016$.

[20] Central Statistical Authority of Ethiopia (CSA) (2012) Population and housing census. Available from http://unstats.un.org/unsd/censuskb20/Attachment489.aspx.

[21] Abrahams PW (2002) Soils: their implications to human health. Sci Tota Environ 291: 1-32.

[22] Desta K, Ashine M, Davey G (2003) "Prevalence of podoconiosis (endemic non-filarial elephantiasis) in Wolaita, Southern Ethiopia". Trop Doct 32: 217- 220.

[23] Kokate K, Purohit AP (1999) Textbook of Pharmacognosy. Page 267-268.

[24] Cooksley VG (1996) In: Aromatherapy-A lifetime guide healing with essential oils, Prentice Hall New Jersey Page 159-195.

[25] Gediya SK, Mistry RB, Patel UK, Blessy M, Jain HN (2011) Herbal Plants: Used as a cosmetics. J Nat Prod Plant Resour 1(1): 24-32.

[26] Silva J, Abebe W, Sousa SM, Duarte VG, Machado MI, Matos FJ (2003) Analgesic and anti-inflammatory effects of essential oils of Eucalyptus. J Ethnopharmacol 89(2-3): 277-283.

[27] Nagpal G, Shah M, Arora N, Shri R, Arya Y (2010) Phytochemical and Pharmacological Aspects of Eucalyptus Genus. Int J Pharmaceutic Sci Res 1:28-36. 